
DEUTSCHE AKADEMIE DER WISSENSCHAFTEN

ZU BERLIN

Forschungsgemeinschaft der naturwissenschaftlichen, technischen und medizinischen Institute

\section{UBER WISSENSCHAFTLICHE GRUNDLAGEN DER MODERNEN TECHNIK}

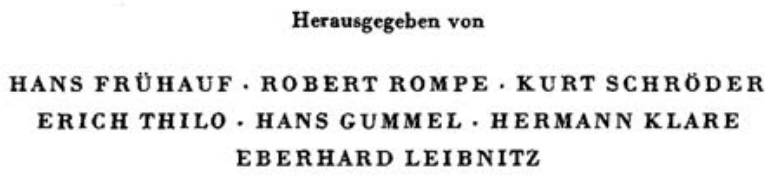

REIHE A: TAGUNGEN

BAND I

ELEKTROTECHNIK UND MASCHINENBAU

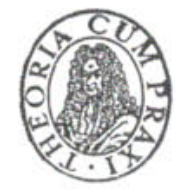

AKADEMIE-VERLAG - BERLIN 


\title{
ELEKTROTECHNIK UND
}

\section{MASCHINENBAU}

\author{
Vorträge,
}

gehalten auf der Konferenz der Forschungsgemeinschaft der Deutschen Akademie der Wissenschaften zu Berlin am 8. und 9.12.1960 in Berlin-Adlershof 


\section{Redaktion:}

Wissenschaftliches Sekretariat der Forschungsgemeinschaft der Deutschen Akademie der Wissenschaften zu Berlin

Pressestelle der Deutschen Akademie der Wissenschaften zu Berlin

Erschienen im Akademie -Verlag GmbH, Berlin W 8, Leipziger Straße 3-4 Copyright 1961 by Akademie -Verlag GmbH

Lizenznummer : $202 \cdot 100 / 529 / 61$

Gesamtherstellung: Druckhaus „Maxim Gorki", Altenburg

Bestellnummer : 2104/A/1/61

Printed in Germany

ES $20 \mathrm{H} 1 / 20 \mathrm{~K} 1$ 
Am 8. und 9. Dezember 1960 veranstaltete die Forschungsgemeinschaft der naturwissenschaftlichen, technischen und medizinischen Institute der Deutschen Akademie der Wissenschaften zu Berlin eine Konferenz „Über wissenschaftliche Grundlagen der modernen Technik - Elektrotechnik und Maschinenbau".

Diese Konferenz und die aus ihr resultierende vorliegende Arbeit sind Ausdruck des Verantwortungsbewußtseins dafür, daß die Akademie bei der Entwicklung und Festigung der wissenschaftlichen Grundlagen der Produktion wirksame Anstrengungen unternimmt.

Der dargebotene Band - Auftakt zu einer Schriftenreihe der Forschungsgemeinschaft der Akademie - setzt sich aus den Vorträgen namhafter Wissenschaftler und Praktiker zusammen, die

zu den Entwicklungstendenzen auf dem Gebiet der Elektronik, zu Fragen moderner mathematischer Methoden in der Elektrotechnik und im Maschinenbau,

zu Problemen moderner physikalischer Analysenmethoden,

zu Gebieten neuer metallischer Werkstoffe für Elektrotechnik und Maschinenbau

sowie zu Kunststoffen mit besonderen physikalischen Eigenschaften

eine für Theorie und Praxis förderliche Auseinandersetzung und Verständigung einleiteten.

Am 21. April 1946 wurde die Sozialistische Einheitspartei Deutschlands durch Vereinigung der Kommunistischen Partei Deutschlands und der Sozialdemokratischen Partei Deutschlands gegründet.

Zur 15. Wiederkehr des Gründungstages der Sozialistischen Einheitspartei Deutschlands lege ich im Auftrage des Vorstandes der Forschungsgemeinschaft der Deutschen Akademie der Wissenschaften zu Berlin diese Arbeit als Würdigung der Leistungen, die für Frieden, Demokratie und Sozialismus von der Partei der geeinten Arbeiterklasse erbracht wurden, vor. 
